\title{
Guest editorial to the special issue: subsurface environmental simulation benchmarks
}

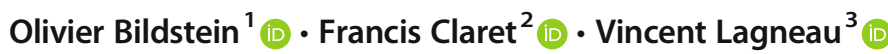

Published online: 26 June 2021

(C) The Author(s) 2021

\section{Objective of the special issue}

This special issue follows the highly successful first issue published in 2015 by Computational Geosciences (Steefel, Yabusaki, and Mayer, editors) [1]. The intent of this second special issue was to follow this initiative and extend the set of well-described benchmark problems that can be used to demonstrate simulator conformance with norms established by the subsurface science and engineering community. This is particularly relevant to reactive transport tools in the context of large-scale and global environmental changes and their global impacts that challenge the subsurface science and engineering community, especially since the subsurface has an important part to play in the energy transition. For example, it may be tempting to use the subsurface for large-scale storage of energy to smooth the intermittent supply of renewable energies, to store anthropogenic carbon dioxide in minerals and geological formations, to enable large-scale hydrogen storage in porous media or to store nuclear waste [1-3]. Providing accurate multi-physical assessments of risk and engineering performance for such technological solutions with far-reaching consequence relies, among others, on numerical models that integrates, in a consistent framework, an increasing amount of scientific knowledge. In this context, since about 30 years, reactive transport modeling (RTM) has established itself at a

Francis Claret

f.claret@brgm.fr

Olivier Bildstein

olivier.bildstein@cea.fr

Vincent Lagneau

vincent.lagneau@mines-paristech.fr

1 Commissariat à l'Energie Atomique et aux Energies Alternatives (CEA), Cadarache, France

2 BRGM, Orleans, France

3 MINES ParisTech, PSL University, Paris, France very powerful tool to account for and quantify the complexity of coupled processes over a long period of time.

\section{Content of the special issue}

As pointed out by Steefel et al. (2015) [4], active topics of research dealing with RTM include the development of pore scale and hybrid, or multiple continua, models to capture the scale dependence of coupled reactive transport processes. In this issue, two benchmarks explore this direction, one focusing on mineral dissolution at the pore scale [5] and the other one testing capability of simulating multicomponent and dual continuum diffusion in bulk and diffuse layer water [6]. For this latter case, a benchmark consists in providing a set of exercises that may be used by code developers to verify their implementation of the Nernst-Planck equation together with the presence of diffuse layer capabilities in their software. In the previous issue, a benchmark was focusing on the assessment of electromigration and species-dependent diffusion in the context of Nernst-Planck equation but without considering charged surfaces [7], while in a second one the need for benchmark exercises to accurately validate numerical implementation of set of equations that are formulated in terms of the Nernst-Planck equation was identified when simulating a column experiment in compacted bentonite with multispecies diffusion and explicit treatment of electrostatic effects [8]. The Nernst-Planck equation allows to consider, at the continuum scale, the influence of a diffuse layer that balances mineral surface charge. This is particularly relevant for nano-porous media such as clay materials that are under consideration for nuclear disposal since the electrostatic properties of clay minerals can play a large role on ion diffusion. While in the bulk porosity electroneutrality prevails, the negative electrostatic potential field at the clay mineral surfaces results in the presence of porosity domains where electroneutrality is not achieved in the aqueous solution: cations are attracted by the surfaces while anions are repulsed from them, resulting in the 
presence of a diffuse layer. This benchmark is not only relevant for clay media but also for other nano-porous media where a surface charge is developed like concrete also widely use as an engineered barrier for many nuclear waste repositories.

At the pore scale [5], the simulation of single-phase flow, reactive transport, and solid geometry evolution compares approaches typically employed for solving pore-scale problems, namely discretization methods, characterization of the fluidsolid interfaces, and moving interface methods as a result of fluid-solid reactions. As opposed to historical approaches that rely on constitutive equations that make it possible to describe the porous medium of interest at the continuum scale, with respect to its macroscopically measurable properties such as permeability, dispersivity, diffusibility, and so on, at the pore scale, the continuum assumption applies to each phase separately and the pore network is fully resolved. The last decade has seen a growing interest in pore scale modeling because they allow for better description of the complex interplay between reactions and transport and solid geometry that can lead to highly nonlinear effect usually poorly captured at the continuum scale.

Such nonlinear effects were tackled in another benchmark, where the impact of mineral dissolution-precipitation reactions on porosity and its coupling on transport was studied [9]. This benchmark considers the advective-diffusive transport of solutes in a complex 2D geometry with the effect of liquid-phase density on liquid flow and advective transport, kinetically controlled dissolution-precipitation reactions causing porosity, permeability, and diffusivity changes; and the formation of a $\mathrm{BaSO}_{4}-\mathrm{SrSO}_{4}$ solid solution. In comparison to the benchmark of Xie et al. [10], published in the previous issue, more complexity have been considered regarding chemistry (solid solution) and transport (density flow). This benchmark is relevant for many applications such as $\mathrm{CO}_{2}$ storage, scaling issues when using geothermal energy, and nuclear waste disposal.

While $\mathrm{CO}_{2}$ storage is envisaged as a method to mitigate greenhouse gas emissions while performing the transition to low-carbon energy, the cycling of organic carbon in soils also plays a major role in controlling atmospheric $\mathrm{CO}_{2}$ and therefore affects global climate change. Having numerical models capable of coupling physics-based models for water flow, as well as solute and gas with state-of-the-art soil organic matter (SOM) degradation models is a key issue. A benchmark for SOM degradation under variably saturated flow conditions was set up in this context [11]. In its highest level of complexity the SOM degradation kinetic networks with reactive transport under variably saturated flow conditions, including $\mathrm{CO}_{2}$ transport from soils to the atmosphere was considered.

Concerning isotopes, two benchmarks were proposed. The first one explores sulfur isotopic fractionation resulting from microbial aqueous sulfate reduction, and iron-sulfide dissolution and precipitation reactions [12] using results from a large, well-characterized column experiment. This benchmark includes reactions previously proposed with the $\mathrm{Cr}$ geochemical system in the previous special issue by Wanner et al. [13] and introduces the influence of mineral reactions and Monod type kinetics for aqueous reactions. Results show a reasonable agreement with experimental results and between codes. An additional benchmark is also proposed on the full account of stable and radioactive carbon isotopes both for stable equilibrium and kinetic fractionation [14]. A series of components lead the authors from modeling equilibrium in batch systems to a full 1D flow path with mass-dependent isotope fractionation due to the oxidation of DOC into DIC by sulfate reduction. In this benchmark, an excellent agreement is obtained between the different codes.

A final benchmark deals with Cs sorption which play a major role on Cs migration in clay formations [15]. The motivation of this benchmark partly stems from the complex, non-linear character of Cs retention (multicomponent, multisite ion exchange models) which affects the computing time, especially when modeling large-scale systems such as radioactive waste repositories. A first component of the benchmark was constructed with this mechanistic approach and yielded good agreement between the different codes. A second component proposed an alternative approach using a much faster single-species reactive transport (Cs) with a with sorption isotherm taking the form of a look-up table accounting for the dependency of retention with regard to Cs concentration in solution only. In this case, the comparison performed provides a fairly good agreement between codes and also when considering the two approaches with the same code.

\section{Emerging benchmarking opportunities}

Machine learning is considered as a recent disruptive technology in the field of reactive transport and will possibly unlock the next generation of simulation that require high demanding CPU time [16]. The high computing cost associated with chemical equilibrium calculations is considering as the most demanding one in comparison to fluid flow or heat transfer and to circumvent this issue the use of surrogate model is promising $[17,18]$. In the future, having a benchmark that tackle this issue will be very useful.

Finally, multiphase multicomponent reactive transport is rapidly developing in the subsurface environment simulation community. The new physics brings additional nonlinearities in flow and geochemistry related equations, as well as vaporliquid equilibrium [19]. The numerical complexity, and the numerous potential applications (gas storage, corrosion gas production, oxygen availability in the vadose zone...), stress the need for careful RTM code comparison for accurate modeling. 
Acknowledgments The authors would like to acknowledge the funding of the Agence Nationale de la Recherche (ANR) under Contract No. ANR-18-CE05-0035-01, the European Union's Horizon 2020 research and innovation program under Grant Agreement No. 847593 and the Andra (the French waste management agency) under the CEA/ANDRA collaborative agreement $n^{\circ} \mathrm{CHR}$.

Open Access This article is licensed under a Creative Commons Attribution 4.0 International License, which permits use, sharing, adaptation, distribution and reproduction in any medium or format, as long as you give appropriate credit to the original author(s) and the source, provide a link to the Creative Commons licence, and indicate if changes were made. The images or other third party material in this article are included in the article's Creative Commons licence, unless indicated otherwise in a credit line to the material. If material is not included in the article's Creative Commons licence and your intended use is not permitted by statutory regulation or exceeds the permitted use, you will need to obtain permission directly from the copyright holder. To view a copy of this licence, visit http://creativecommons.org/licenses/by/4.0/.

\section{References}

1. Heinemann, N., Alcalde, J., Miocic, J.M., Hangx, S.J.T., Kallmeyer, J., Ostertag-Henning, C., Hassanpouryouzband, A., Thaysen, E.M., Strobel, G.J., Schmidt-Hattenberger, C., Edlmann, K., Wilkinson, M., Bentham, M., Stuart Haszeldine, R., Carbonell, R., Rudloff, A.: Enabling large-scale hydrogen storage in porous media - the scientific challenges. Energy Environ. Sci. 14(2), 853-864 (2021). https://doi.org/10.1039/D0EE03536J

2. Kelemen, P., Benson, S.M., Pilorgé, H., Psarras, P., Wilcox, J.: An Overview of the Status and Challenges of CO2 Storage in Minerals and Geological Formations. Frontiers in Climate 1(9) (2019). doi: https://doi.org/10.3389/fclim.2019.00009

3. Kabuth, A., Dahmke, A., Beyer, C., Bilke, L., Dethlefsen, F., Dietrich, P., Duttmann, R., Ebert, M., Feeser, V., Görke, U.-J., Köber, R., Rabbel, W., Schanz, T., Schäfer, D., Würdemann, H., Bauer, S.: Energy storage in the geological subsurface: dimensioning, risk analysis and spatial planning: the ANGUS+ project. Environ. Earth Sci. 76(1), 23 (2016). https://doi.org/10.1007/ s12665-016-6319-5

4. Steefel, C.I., Appelo, C.A.J., Arora, B., Jacques, D., Kalbacher, T., Kolditz, O., Lagneau, V., Lichtner, P.C., Mayer, K.U., Meeussen, J.C.L., Molins, S., Moulton, D., Shao, H., Simunek, J., Spycher, N., Yabusaki, S.B., Yeh, G.T.: Reactive transport codes for subsurface environmental simulation. Comput. Geosci. 19(3), 445-478 (2015). https://doi.org/10.1007/s10596-014-9443-x

5. Molins, S., Soulaine, C., Prasianakis, N.I., Abbasi, A., Poncet, P., Ladd, A.J.C., Starchenko, V., Roman, S., Trebotich, D., Tchelepi, H.A., Steefel, C.I.: Simulation of mineral dissolution at the pore scale with evolving fluid-solid interfaces: review of approaches and benchmark problem set. Comput. Geosci. (2020). https://doi. org/10.1007/s10596-019-09903-x

6. Tournassat, C., Steefel, C.I.: Modeling diffusion processes in the presence of a diffuse layer at charged mineral surfaces: a benchmark exercise. Comput. Geosci. (2019). https://doi.org/10.1007/ s10596-019-09845-4

7. Rasouli, P., Steefel, C.I., Mayer, K.U., Rolle, M.: Benchmarks for multicomponent diffusion and electrochemical migration. Comput. Geosci. 19(3), 523-533 (2015). https://doi.org/10.1007/s10596015-9481-z
8. Alt-Epping, P., Tournassat, C., Rasouli, P., Steefel, C.I., Mayer, K.U., Jenni, A., Mäder, U., Sengor, S.S., Fernández, R.: Benchmark reactive transport simulations of a column experiment in compacted bentonite with multispecies diffusion and explicit treatment of electrostatic effects. Comput. Geosci. 19(3), 535-550 (2015). https://doi.org/10.1007/s10596-014-9451-x

9. Poonoosamy, J., Wanner, C., Alt Epping, P., Águila, J.F., Samper, J., Montenegro, L., Xie, M., Su, D., Mayer, K.U., Mäder, U., Van Loon, L.R., Kosakowski, G.: Benchmarking of reactive transport codes for 2D simulations with mineral dissolution-precipitation reactions and feedback on transport parameters. Comput. Geosci. (2018). https://doi.org/10.1007/s10596-018-9793-x

10. Xie, M., Mayer, K.U., Claret, F., Alt-Epping, P., Jacques, D., Steefel, C., Chiaberge, C., Simunek, J.: Implementation and evaluation of permeability-porosity and tortuosity-porosity relationships linked to mineral dissolution-precipitation. Comput. Geosci. 19(3), 655-671 (2015). https://doi.org/10.1007/s10596-014-9458-3

11. Jia, M., Jacques, D., Gérard, F., Su, D., Mayer, K.U., Šimůnek, J.: A benchmark for soil organic matter degradation under variably saturated flow conditions. Comput. Geosci. (2019). https://doi. org/10.1007/s10596-019-09862-3

12. Cheng, Y., Arora, B., Șengör, S.S., Druhan, J.L., Wanner, C., van Breukelen, B.M., Steefel, C.I.: Microbially mediated kinetic sulfur isotope fractionation: reactive transport modeling benchmark. Comput. Geosci. (2020). https://doi.org/10.1007/s10596-02009988-9

13. Wanner, C., Druhan, J.L., Amos, R.T., Alt-Epping, P., Steefel, C.I.: Benchmarking the simulation of $\mathrm{Cr}$ isotope fractionation. Comput. Geosci. 19(3), 497-521 (2015). https://doi.org/10.1007/s10596014-9436-9

14. Druhan, J.L., Guillon, S., Lincker, M., Arora, B.: Stable and radioactive carbon isotope partitioning in soils and saturated systems: a reactive transport modeling benchmark study. Comput. Geosci. (2020). https://doi.org/10.1007/s10596-020-09937-6

15. Águila, J.F., Montoya, V., Samper, J., Montenegro, L., Kosakowski, G., Krejci, P., Pfingsten, W.: Modeling cesium migration through Opalinus clay: a benchmark for single- and multispecies sorption-diffusion models. Comput. Geosci. (2021). https:// doi.org/10.1007/s10596-021-10050-5

16. Leal, A.M.M., Kyas, S., Kulik, D.A., Saar, M.O.: Accelerating reactive transport modeling: on-demand machine learning algorithm for chemical equilibrium calculations. Transp. Porous Media. 133(2), 161-204 (2020). https://doi.org/10.1007/s11242020-01412-1

17. Laloy, E., Jacques, D.: Emulation of CPU-demanding reactive transport models: a comparison of Gaussian processes, polynomial chaos expansion, and deep neural networks. Comput. Geosci. 23(5), 1193-1215 (2019). https://doi.org/10.1007/s10596-01909875-y

18. Prasianakis, N.I., Haller, R., Mahrous, M., Poonoosamy, J., Pfingsten, W., Churakov, S.V.: Neural network based process coupling and parameter upscaling in reactive transport simulations. Geochim Cosmochim Ac. 291, 126-143 (2020). https://doi.org/ 10.1016/j.gca.2020.07.019

19. Sin, I., Corvisier, J.: Multiphase multicomponent reactive transport and flow modeling. Rev. Mineral. Geochem. 85(1), 143-195 (2019). https://doi.org/10.2138/rmg.2019.85.6

Publisher's note Springer Nature remains neutral with regard to jurisdictional claims in published maps and institutional affiliations. 